\title{
Dual Channel Monopulse Automatic Phase Calibration Method
}

\author{
Xinfeng Fan ${ }^{1,}$,, Yongming $\mathrm{Nie}^{1, b^{*}}$ and Xin Ding ${ }^{1, c}$ \\ ${ }^{1}$ China Satellite Maritime Tracking and Control Department, Jiangyin, Jiangsu, \\ China \\ ayimonie@163.com, bnwy1986@163.com, ${ }^{\mathrm{c} 290505026 @ q q . c o m}$ \\ * The corresponding author
}

Keywords: Tracking; Phase calibration; Sum and difference channels; Cross coupling

\begin{abstract}
Combining the target flight and acquisition characteristics with tracking process, a phase calibration method is proposed through theoretical analysis of dual channel monopulse tracking system angular error demodulation principle. The key of that is a new real-time correction phase and real time cross coupling synthetic elimination scheme, which can quickly complete the phase calibration based on the method of radar terminal demodulation output cross coupling error angle voltage. It can use static beacons to achieve phase calibration and also can be in in the process of tracking flying object realizing real-time dynamic phase correction.
\end{abstract}

\section{Introduction}

In the maritime tracking and control system, the angle self tracking is the pre-condition for the completion of the measurement and control task [1,2]. At present, most of the angle tracking systems adopts the dual channel monopulse tracking scheme, which is of the properties such as high precision, hig data speed, far distance and strong anti-interference ability [3, 4]. However, the phase difference between the sum and difference channels should be calibrated to obtain the angle error voltage correctly. There are many methods to resolve this problem [5-6]. However, considering the shipborne complex dynamic conditions, new designation should be taken into account to be suitable for shipborne ladar [7-8]. Traditional phase standard calibration methods need antenna being strict alignment with target firstly $[9,10]$, then manual operating antenna for partial azimuth and elevation deviation. By way of automatic phase shifter and related operations, the channel transmission delay and the antenna directional sensitivity are obtained. The method is time consuming and is difficult to guarantee the antenna alignment with target strictly on the ship borne floating platform radar. In this manuscript, a phase calibration method is proposed, which can quickly complete the phase calibration based on the method of radar terminal demodulation output cross coupling error angle voltage. It can use static beacons to achieve phase calibration and also can be in in the process of tracking flying object realizing real-time dynamic phase correction. 


\section{Theory Analysis}

Dual Channel Monopulse Tracking Scheme. Taking the left-hand circular polarization signal as an example, the angular error demodulation formula is as follows.

$$
\left[\begin{array}{l}
U_{A z} \\
U_{E l}
\end{array}\right]=\left[\begin{array}{ll}
\cos \Delta \varphi & \sin \Delta \varphi \\
\sin \Delta \varphi & \cos \Delta \varphi
\end{array}\right] \times\left[\begin{array}{c}
U_{A z}^{\prime} \\
U_{E l}^{\prime}
\end{array}\right]
$$

Where $U_{A z}$ is output azimuth angle error voltage, $U_{E l}$ is output elevation angle error voltage, $\Delta \varphi$ is phase difference between two channels, $U_{A z}^{\prime}$ is real azimuth angle error voltage, $U_{E t}^{\prime}$ is real elevation angle error voltage. When $\Delta \varphi$ equals to zero, the azimuth and pitch angle error of the radar terminal strictly equals to the angle error of the actual target. When $\Delta \varphi$ does not equal to zero, azimuth and elevation angle error demodulation output radar terminal voltage does not strictly equal to the actual target deviation of the antenna axis azimuth and pitching angle error voltage. So cross coupling transformation relationship between them should be eliminated.

Real Time Phase Correction Method. For static target, the initial state is angle error voltage is zero, so the antenna aligns with target strictly. Azimuth and elevation angle errors are as follows.

$$
\left\{\begin{array}{l}
U_{A z}=U_{A z}^{\prime}(t) \times \cos (\Delta \varphi) \\
U_{E l}=U_{A z}^{A}(t) \times \sin (\Delta \varphi)
\end{array}\right.
$$

So the expression of $\Delta \varphi$ can be written as follows.

$$
\begin{cases}\Delta \varphi=\operatorname{arctg}\left(\left|\frac{U_{E l}}{U_{A z}}\right|\right) & U_{A z}>0, U_{E l}>0 \\ \Delta \varphi=2 \pi-\operatorname{arctg}\left(\left|\frac{U_{E l}}{U_{A z}}\right|\right) & U_{A z}>0, U_{E l}<0 \\ \Delta \varphi=\pi-\operatorname{arctg}\left(\left|\frac{U_{E l}}{U_{A z}}\right|\right) & U_{A z}<0, U_{E l}>0 \\ \Delta \varphi=\pi+\operatorname{arctg}\left(\left|\frac{U_{E l}}{U_{A z}}\right|\right) & U_{A z}<0, U_{E l}<0\end{cases}
$$

According to the theoretical analysis above, in the initial state antenna power shaft alignment with target, if antenna is manually pulled partial angle range, radar terminal actual demodulation of pitch angle error and azimuth error curve is a straight line with slope of $\operatorname{tg}(\Delta \varphi)$. The channel transmission delay difference can be obtained according to the slope of the line.

For static target, the initial state is angle error voltage is not zero, so the antenna does not align with target. Azimuth and elevation angle errors are as follows. 


$$
\left\{\begin{array}{l}
U_{A z O}=U_{A z O}^{\prime} \times \cos (\Delta \varphi)+U_{E l O}^{\prime} \times \sin (\Delta \varphi) \\
U_{E l O}=U_{A z O}^{\prime} \times \sin (\Delta \varphi)+U_{E l O}^{\prime} \times \cos (\Delta \varphi)
\end{array}\right.
$$

After detail theretical deducing which is no longer given, the expression of $\Delta \varphi$ can be written as follows.

$$
\begin{cases}\Delta \varphi=\operatorname{arctg}\left(\left|\frac{\Delta U_{E l}}{\Delta U_{A z}}\right|\right) & \Delta U_{A z}>0, \Delta U_{E l}>0 \\ \Delta \varphi=2 \pi-\operatorname{arctg}\left(\left|\frac{\Delta U_{E l}}{\Delta U_{A z}}\right|\right) & \Delta U_{A z}>0, \Delta U_{E l}<0 \\ \Delta \varphi=\pi-\operatorname{arctg}\left(\left|\frac{\Delta U_{E l}}{\Delta U_{A z}}\right|\right) & \Delta U_{A z}<0, \Delta U_{E l}>0 \\ \Delta \varphi=\pi+\operatorname{arctg}\left(\left|\frac{\Delta U_{E l}}{\Delta U_{A z}}\right|\right) & \Delta U_{A z}<0, \Delta U_{E l}<0\end{cases}
$$

The same as the situation above, the channel transmission delay difference can be obtained according to the slope of the line. For moving target, the analyzing method is the same, so the detail analysis is no longer given. Just the application of the theory above is demostrated as follows.

\section{Realization of Phase Caliberation and Cross Coupling Elimination}

The real-time phase calibration module receives the azimuth and elevation angle errors from the radar terminal combing with the theoretical refernce and timing information, channel transmission delay difference is real-time obtained. The principle process is shown in Fig. 1(a).

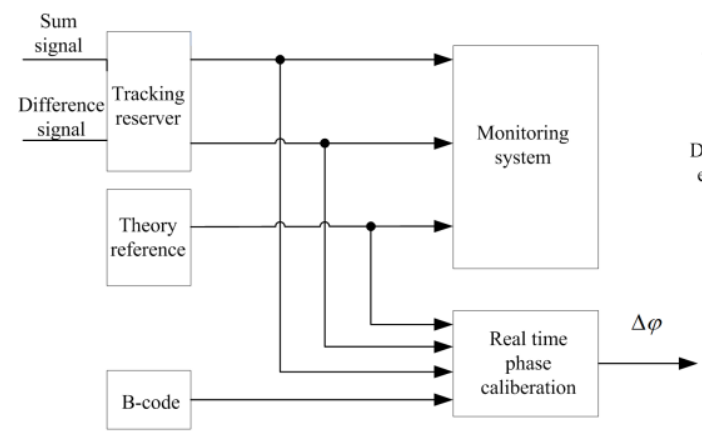

(a)

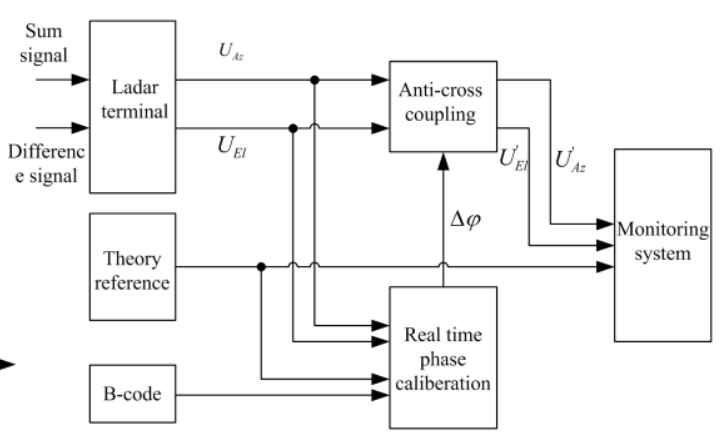

(b)

Figure 1. Phase caliberation and cross coupling elimination diagram, (a) is phase caliberation schematic and (b) is cross coupling elimination diagram

When $\Delta \varphi$ is not equal to zero, there is cross coupling. In order to elimate it, making the demodulation target angle error being exactly equal to the actual target angle error, a method is to eliminate differential channel phase difference at front of radar terminal, which real time introduces phase calibration module into closed 
loop servo tracking process, not only achieving real-time correctiing of the phase, but also can greatly improve the servo angle tracking system adaptive ability.

\section{Conclusions}

A method of automatic phase correction proposed here can be used to realize the dynamic calibration with the tower or during target tracking process. On this basis, a new real-time cross coupling elimination scheme is put forward, which through data processing way to eliminate the radar terminal demodulation output cross coupling between the servo control azimuth and elevation error voltage. The scheme not only realizes the tracking system in the moving target, but also the angle servo tracking system has the sum and difference channel phase transmission delay adaptive ability, which will improve the reliability of the system and enhance the system repair ability.

\section{References}

[1]. B. Redman, W. Ruff, B. Stann and et al. Anti-ship missile tracking with a chirped AM ladar Update: design, model predictions, and experimental results, Proc Spie, 2005, 5791:330-341.

[2]. Z. LI, J. LI and Q. Wang. Tracking algorithm of ladar, Infrared \& Laser Engineering, 2006, 35(6):696-699.

[3]. Incorvaia, T. Cadili, A. Genovese and et al. Monopulse tracking feed system for next Q/V-band applications, European Conference on Antennas \& Propagation, 2014:889-893.

[4]. Cong, Y. Qu, Y. Zhang. Analytic performance of monopulse spread spectrum tracking system in multiple-target, Scenario Journal of Physics Conference Series, 2016, 679-683.

[5]. Y. Zhi, J. Sun, P Hou and et al. Phase error correction by phase differential algorithm for synthetic aperture imaging ladar, Proc Spie, 2012, 8520(4): 434-437.

[6]. Z. Hua, H. Li and Y.Gu. A hybrid method for synthetic aperture ladar phase-error compensation, International Symposium on Photoelectronic Detection and Imaging 2009, 7382.

[7]. X. Wu, J. Zhao, W. Wang and et al. Design and Implementation of a Multi-Band and Multi-Target Maritime Tracking and Control Simulator, Journal of Spacecraft Tt \& C Technology, 2011, 06: 107-110.

[8]. H. Wang, B. Liu, C. Xue and et al. Strategy and Application of Command and Display Optimization in Maritime Tracking and Controlling Mission, Ordnance Industry Automation, 2014, 08: 213-216. 
[9]. B. Gao and J. D. Mathews. Phase and pattern calibration of the Jicamarca Radio Observatory radar using satellites, Science \& Mathematics, 2015, 446(4):3416-3426.

[10]. X. Zhou, H. Wang, Y. Cheng and et al. Sparse Auto-Calibration for Radar Coincidence Imaging with Gain-Phase Errors, Sensors, 2015, 15(11):27611-27624. 